\title{
Clustering Matrix Sequences Based on the Iterative Dynamic Time Deformation Procedure
}

\author{
Zhengbing Hu \\ School of Educational Information Technology, Central China Normal University, Wuhan, China \\ E-mail: hzb@mail.ccnu.edu.cn \\ Sergii V. Mashtalir \\ Kharkiv National University of Radio Electronics, Kharkiv, Ukraine \\ E-mail: sergii.mashtalir@ nure.ua \\ Oleksii K. Tyshchenko \\ Institute for Research and Applications of Fuzzy Modeling, CE IT4Innovations, \\ University of Ostrava, Ostrava, Czech Republic \\ E-mail: lehatish@gmail.com \\ Mykhailo I. Stolbovyi \\ Kharkiv National University of Radio Electronics, Kharkiv, Ukraine \\ E-mail: mykhailo.stolbovyi@nure.ua
}

Received: 03 April 2018; Accepted: 15 May 2018; Published: 08 July 2018

\begin{abstract}
The techniques of Dynamic Time Warping (DTW) have shown a great efficiency for clustering time series. On the other hand, it may lead to sufficiently high computational loads when it comes to processing long data sequences. For this reason, it may be appropriate to develop an iterative DTW procedure to be capable of shrinking time sequences. And later on, a clustering approach is proposed for the previously reduced data (by means of the iterative DTW). Experimental modeling tests were performed for proving its efficiency.
\end{abstract}

Index Terms-Time Series, Segmentation, Clustering, Video Stream, Proximity Measure, Dynamic Time Warping.

\section{INTRODUCTION}

The development of information technology has led to a rapid growth in volumes of data processed. There is a sufficient amount of approaches for text data, but the multimedia analysis, and especially a technique used for a case when it is necessary to take into account the data content (content-based approaches) has not been widely developed yet. This problem is especially typical for video data processing due to the maximum hardness of the analysis process in a time-limited environment [1-4].

Moreover, the complexity is rather high not only due to big data volumes, but also due to their insufficient structuredness, and therefore there are whole sets of scenes (shots) within a single video sequence that are weakly correlated or not correlated at all with each other. At the same time, the correlation within the same shot remains rather strong [5]. So if you need to solve some problems, for example, to search for a content in video data, there are serious time limits. To accelerate the analysis in the domain of video sets, some preliminary processing is required which allows speeding up the process considerably. Since it can be an offline video clustering/segmentation procedure to represent a video as a set of content-homogeneous segments [6-18]. Given the fact that any video sequence can be represented in the form of a sequence of frames, it is advisable to use the multidimensional (matrix) time series analysis [19, 20].

The aim of clustering time series holds a specific place in the whole domain of Data Mining [21-23]. For the purposes of time series clustering, there are multiple methods to have been proposed and examined which are distinct from each other by the initial assumptions, by the mathematical apparatus applied, and by the computational complexity of their actualization [8-11]. In particular, this problem takes a peculiar place in the analysis of video sequences where most of the known techniques lose their efficiency. It can be justified in the first instance by the fact that high demands on performance step forward in the context of large volumes of processed data, and actual clustering objects are matrix time series $X_{1}, \ldots, X_{q}, X_{r}, \ldots, X_{Q}$, and each one contains $N_{1}, \ldots, N_{q}, N_{r}, \ldots, N_{Q}$ observations in such a way that $X_{r}=\left\{x_{r}(1), x_{r}(2) \ldots, x_{r}(k), \ldots, x_{r}\left(N_{r}\right)\right\} \quad, \quad$ and every individual observation is revealed as a $(n \times m)$ - matrix $X_{r}(k)=\left\{x_{r i_{1} i_{2}}(k)\right\} i_{1}=1,2, \ldots, n, i_{2}=1,2, \ldots, m$.

At the same time, the major challenges to appear while 
clustering time series are determined by the fact that these series are distinguished by different lengths and mainly not so much by the high dimensionality of the data being processed. For instance, if a series $X_{q}$ stores $N_{q}$ $(n \times m)$-matrices of video snapshots, then a series $X_{r}$ contains $N_{r}$ matrices of the same dimensionality subsequently that doesn't make it possible to use a residual quantity $\Delta_{q r}=X_{q}-X_{r}$ to determine similarity between them as well as to take an advantage of the known norms of this residual accordingly. As an alternative way to traditional norms, it can be some certain proximity rate [11, 24-27]. A method of the Dynamic Time Warping (DTW [28-36]) may be one of the aforesaid proximity measures. This technique is chiefly grounded on the Euclidean norm to be calculated between two vectors of observations. It is conceivable that a spherical norm (which is an extension of the Euclidean one) may be used in the event of matrix observations.

In such a way, if two sequences $X_{q}$ and $X_{r}$ are given with the property that $X_{q}=\left\{x_{q}(1), x_{q}(2) \ldots, x_{q}(k), \ldots, x_{q}\left(N_{q}\right)\right\} \quad$ and $X_{r}=\left\{x_{r}(1), x_{r}(2) \ldots, x_{r}(l), \ldots, x_{r}\left(N_{r}\right)\right\}, N_{q} \neq N_{r}$, it is possible to employ the DTW method for estimation of their similarity or dissimilarity. For that to happen, let's force into application a $\left(N_{q} \times N_{r}\right)$-matrix of distances with elements $d\left(x_{q}(k), x_{r}(l)\right)=\left(\operatorname{Sp}\left(x_{q}(k)-x_{r}(l)\right)\left(x_{q}(k)-x_{r}(l)\right)^{T}\right)^{1 / 2}$.

«warping path» is defined by this matrix to be determined by a sequence of elements

$$
\begin{aligned}
& W=\left\{w_{1}, w_{2}, \ldots, w_{L}\right\}, w_{t}=d\left(x_{q}(k), x_{r}(l)\right)_{t}, \\
& \max \left(N_{q}, N_{r}\right) \leq L<N_{q}+N_{r}+1, t=1,2, \ldots, L .
\end{aligned}
$$

This being said, the smaller it is, the closer (similar) $X_{q}$ and $X_{r}$ are to each other. This path is governed by minimization of a criterion

$$
\operatorname{DTW}\left(X_{q}, X_{r}\right)=\min _{W}\left\{\frac{1}{L} \sum_{t=1}^{L} d\left(x_{q}(k), x_{r}(l)\right)_{t}\right\}
$$

to be implemented through a recurrent procedure of the dynamic programming

$$
\begin{aligned}
& D(k, l)=d\left(x_{q}(k), x_{r}(l)\right)+ \\
& +\min \{D(k, l-1), D(k-1, l), D(k-1, l-1)\}
\end{aligned}
$$

where $D(k, l)$ denotes cumulative distances between elements of the sequences and $x_{q}(k), x_{r}(l)$.

Apparently,

$$
\operatorname{DTW}\left(X_{q}, X_{r}\right)=D\left(N_{q}, N_{r}\right) .
$$

The approach to clustering time series in terms of DTW affirmed it to be effective for solving a set of classical problems [11] (for both a one-dimensional case and a vector one). Although it's fairly obvious that it loses its effectuality in the event of sufficiently long matrix sequences, since it may require high computational efforts.

The remainder of this paper is organized as follows. Section 2 describes a modified iterative dynamic time warping method. Section 3 is devoted to clustering reduced matrix time series. Section 4 presents an experimental research of the work. Conclusions and future work are given in the final section.

\section{A MODIFIED ITERATIVE DYNAMIC TIME WARPING METHOD}

An idea of the Iterative Dynamic Time Warping method consists in reduction of initial time series [37]. For this purpose, the time series analyzed are primarily divided into four sections, and the time series is replaced by its average values in each section. Thus, the time series $X_{q}$ and $X_{r}$ are replaced by the sequences $\bar{X}_{q}$ and $\bar{X}_{r} \quad$ where each one embodies four segments $\bar{x}_{q}(1), \bar{x}_{q}(2), \bar{x}_{q}(3), \bar{x}_{q}(4) \quad$ and $\bar{x}_{r}(1), \bar{x}_{r}(2), \bar{x}_{r}(3), \bar{x}_{r}(4) \quad$ correspondingly. Closeness between two series $\bar{X}_{q}$ and $\bar{X}_{r}$ is further estimated according to a $(4 \times 4)-$ matrix of distances, with that

$$
\operatorname{DTW}\left(\bar{X}_{q}, \bar{X}_{r}\right)=\operatorname{DTW}(4,4)
$$

If the results obtained don't meet the requirements, every segment $\bar{x}_{q}(k), \bar{x}_{r}(k)$ is halved, and the distance is estimated again

$$
\operatorname{DTW}\left(\bar{X}_{q}, \bar{X}_{r}\right)=\operatorname{DTW}(8,8)
$$

The process of halving segments goes underway until the required accuracy of the result is obtained. That said, an amount of analyzed segments grows at an exponential rate.

From the computational point of view, it is much easier to split the original series in such a way that a number of sections grows in an arithmetic progression [2, 38, 39]. Thereby, there are sequences obtained $\bar{X}_{1}, \ldots, \bar{X}_{q}, \bar{X}_{r}, \ldots, \bar{X}_{Q}$ as a result of the reduction procedure, where each one embodies $S$ regions $\bar{X}_{q}=\left\{\bar{x}_{q}(1), \bar{x}_{q}(2) \ldots, \bar{x}_{q}(\mathrm{~S})\right\}, \bar{X}_{r}=\left\{\bar{x}_{r}(1), \bar{x}_{r}(2) \ldots, \bar{x}_{r}(\mathrm{~S})\right\}$. Although the series $\bar{X}_{q}$ and $\bar{X}_{r}$ encapsulate an equal number of regions, it is unacceptable to apply the spherical norm $D\left(\bar{X}_{q}, \bar{X}_{r}\right)=\left(\operatorname{Sp}\left(\bar{X}_{q}-\bar{X}_{r}\right)\left(\bar{X}_{q}-\bar{X}_{r}\right)^{T}\right)^{1 / 2}$ to estimate a distance between them, since regions of both series are characterized by different lengths. 
In a related move, a modification of the assessment (1) can be utilized in the view of

$$
\operatorname{DTW}\left(\bar{X}_{q}, \bar{X}_{r}\right)=D(\mathrm{~S}, S)_{\xi}=\bar{D}\left(\bar{X}_{q}, \bar{X}_{r}\right)
$$

Understandably, any other esteem (traditionally exploited for analysis of time sequences [2]) can be involved instead of the traditional arithmetic mean for each segment of the time series.

\section{Clustering Reduced MATRIX TIME SERIES}

Strictly speaking, any well-known clustering algorithm may be theoretically used for solving the clustering problem. And a clustering metric that designates closeness of the series processed is replaced by the proximity measure (2). With respect to time series, the most popular and effective methods are prototype-based procedures [8-11], where means, medians, and medoids are commonly used as centroids. In [38, 39], precisely these prototypes were utilized for the analysis of video sequences. In addition to the above, if the processed array of video sequences has to be split into $m$ clusters $C l_{1}, C l_{2}, \ldots, C l_{j}, \ldots, C l_{m}$ with centroids $c_{1}, c_{2}, \ldots, c_{j}, \ldots, c_{m}$, a rule of assignment of the series $\bar{X}_{q}$ to the cluster $C l_{j}$ may be put down like [38]

$$
\bar{X}_{q} \in C l_{j} \quad \text { if } \quad \bar{D}\left(\bar{X}_{q}, c_{j}\right)<\bar{D}\left(\bar{X}_{q}, c_{f}\right) \quad \text { for } \quad \text { all }
$$

$q=1,2, \ldots, Q ; j, f=1,2, \ldots, m$.

Initial centroids $c_{j}, c_{f}$ are chosen in a quite arbitrarily way. The clustering process is particularly a sequence of iterations, where centroids' coordinates are being recounted.

For instance, the objective function

$$
\mathrm{E}\left(\bar{X}_{q}, c_{j}\right)=\sum_{q=1}^{Q} \sum_{j=1}^{m} u_{j}(q) \bar{D}^{2}\left(\bar{X}_{q}, c_{j}\right)
$$

is minimized in the process of adjusting centroids in the case of the $k$-means clustering.

And centroids based on mean values are recalculated at every iteration of the procedure according to a relation

$$
c_{j}=\frac{1}{N_{j}} \sum_{\bar{X}_{q} \in C l_{j}} \bar{X}_{q}=\frac{\sum_{q=1}^{Q} u_{j}(q) \bar{X}_{q}}{\sum_{q=1}^{Q} u_{j}(q)}
$$

where $u_{j}(q)$ marks a crisp membership function for the time series to the $j$-th cluster. In this case,

$$
u_{j}(q)=\left\{\begin{array}{l}
1 \text { if } \bar{X}_{q} \in C l_{j} \\
0 \text { otherwise }
\end{array}\right.
$$

Along with that, it is mentioned in [8-11] that the methods grounded on average medians are extremely sensitive to an ineffectual choice of initial centroids in such a way that the clustering process may require a larger number of iterations which is completely unacceptable when it comes to processing large arrays of video information.

Another possible choice is the clustering procedure [41, 42] based on application of harmonic means, in which case a harmonic mean may be presented as

$$
\bar{x}=\frac{Q}{\sum_{q=1}^{Q} x^{-1}(q)}
$$

for a sequence of scalar observations $x_{1}, \ldots, x_{Q}$.

The algorithm offered by B. Zhang and co-authors [40, 41] for an array of vector observations can be updated for clustering matrix objects $\bar{X}_{1}, \ldots, \bar{X}_{q}, \bar{X}_{r}, \ldots, \bar{X}_{Q}$, where each $\bar{X}_{q}$ has a dimensionality of $(n \times m s)$, and a distance between $\bar{X}_{q}$ and a centroid $c_{j}$ is estimated with the help of $\bar{D}\left(\bar{X}_{q}, c_{j}\right)$ according to the expression (2). Upon that, an objective function

$$
\mathrm{E}\left(\bar{X}_{q}, c_{j}\right)=\sum_{q=1}^{Q} \frac{m}{\sum_{j=1}^{m} \frac{1}{\bar{D}^{2}\left(\bar{X}_{q}, c_{j}\right)}}
$$

is minimized during data processing. The mentioned objective function is based on the distance $\bar{D}^{2}\left(\bar{X}_{q}, c_{j}\right)$ just like the function (3).

Minimizing the function (5) by the matrix $c_{j}$, it leads to the estimate

$$
c_{j}=\frac{\sum_{q=1}^{Q} \bar{D}^{-4}\left(\bar{X}_{q}, c_{j}\right)\left(\sum_{j=1}^{m} \bar{D}^{-2}\left(\bar{X}_{q}, c_{j}\right)\right)^{-2} \bar{X}_{q}}{\sum_{q=1}^{Q} \bar{D}^{-4}\left(\bar{X}_{q}, c_{j}\right)\left(\sum_{j=1}^{m} \bar{D}^{-2}\left(\bar{X}_{q}, c_{j}\right)\right)^{-2}},
$$

which is also figured out as a result of a set of iterations (similarly to the expression (4)), although their quantity does not largely depend on initial conditions [10,11]. The authors are introducing the modification (6) in the view of

$$
c_{j}=\frac{\sum_{q=1}^{Q} \bar{D}^{-\alpha}\left(\bar{X}_{q}, c_{j}\right)\left(\sum_{j=1}^{m} \bar{D}^{-2}\left(\bar{X}_{q}, c_{j}\right)\right)^{-2} \bar{X}_{q}}{\sum_{q=1}^{Q} \bar{D}^{-\alpha}\left(\bar{X}_{q}, c_{j}\right)\left(\sum_{j=1}^{m} \bar{D}^{-2}\left(\bar{X}_{q}, c_{j}\right)\right)^{-2}}
$$

where an argument $\alpha$ has a point close to a fuzzifier value that is traditionally utilized in fuzzy clustering procedures.

It does not look difficult to examine fuzzy updates of the procedures (6) and (7) in terms of the approach covered in [8]. At this point, a fuzzy membership level of 
the $q$-th time series to the $j$-th cluster can be computed in obedience to

$$
u_{j}(q)=\frac{\bar{D}^{-2}\left(\bar{X}_{q}, c_{j}\right)}{\sum_{j=1}^{m} \bar{D}^{-2}\left(\bar{X}_{q}, c_{j}\right)}
$$

for the centroids (6) and

$$
u_{j}(q)=\frac{\left(\bar{D}^{2}\left(\bar{X}_{q}, c_{j}\right)\right)^{\frac{1}{1-\beta}}}{\sum_{j=1}^{m}\left(\bar{D}^{2}\left(\bar{X}_{q}, c_{j}\right)\right)^{\frac{1}{1-\beta}}}
$$

for the expression (7). In this case, $\beta>0$ stands for a fuzzifier magnitude (a fuzzification parameter) to specify borders' smearing between the formed clusters.

It should be noted that obtaining the esteems (6) and (7) is not more complicated than evaluation of the centroids
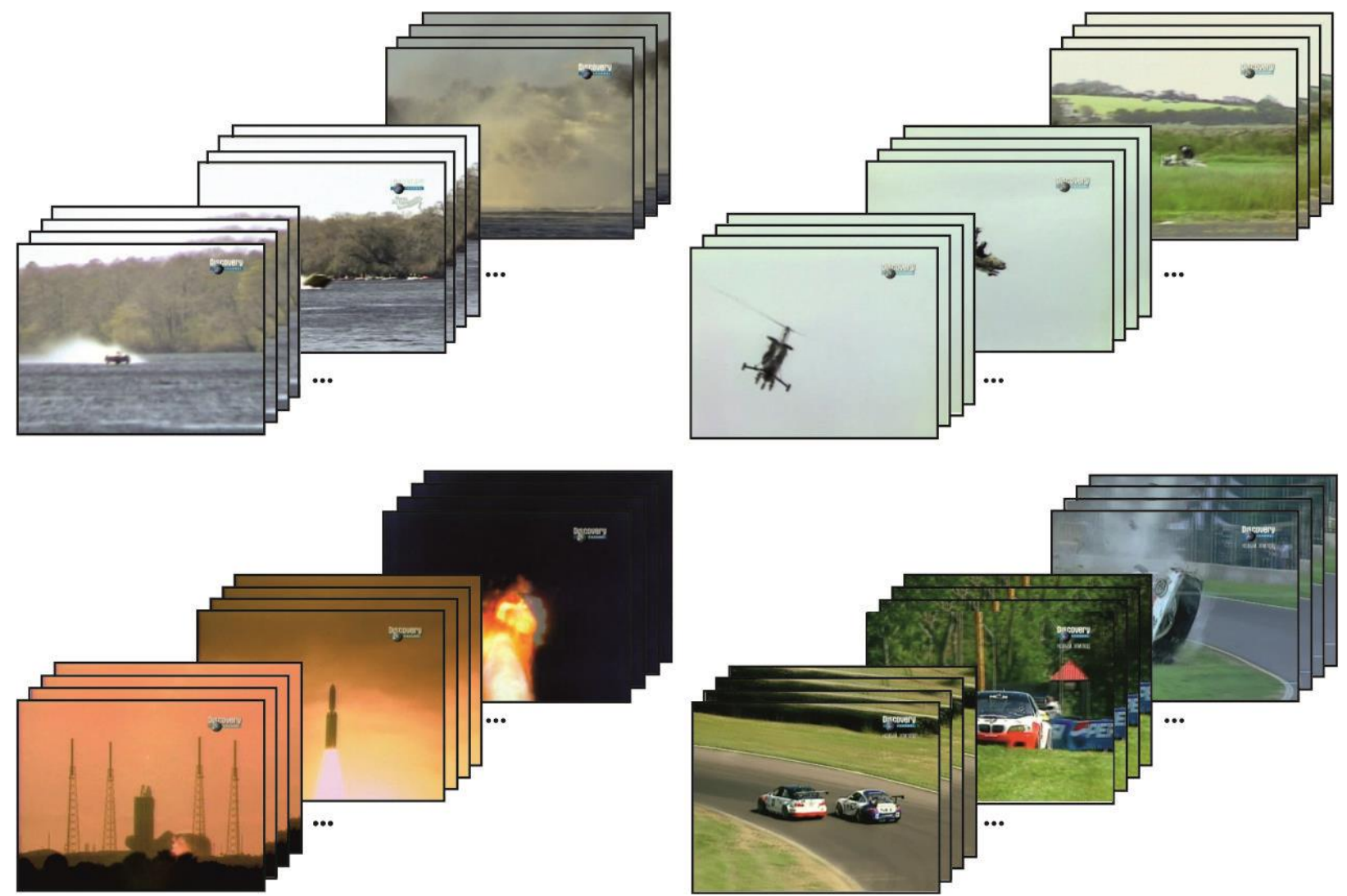

Fig.1. Examples of video sequences presented by a set of frames from the database
Further, for all sequences from the database in an offline mode, the spatio-temporal segmentation of video sequences is implemented. These preliminary calculations are necessary for the early detection of properties' changes in the source data for information retrieval. An example of the spatial segmentation for an initial video sequence is demonstrated in Fig.2. And Fig.3 presents the results of the temporal segmentation. As one in the $k$-means algorithm from a computational standpoint, but it requires fewer iterations.

\section{EXPERIMENTS}

To conduct an experiment for testing the proposed approach, a video clip database was created on the grounds of the series "Destroyed in Seconds" by the Discovery Chanel. A choice of such a movie clip was specified by a fast-changing video and the presence of some scenes with similar characteristics within a single video sequence. Fig.1 shows several video sequences from the database. In this case, as an example, several frames with similar characteristics are shown for some segments of each video sequence that display the processes occurring in the video from the usual shooting of the event to the disaster. can see, peaks (in Fig.3) make it possible to determine changes (it can be seen from the provided video frames) in a homogeneous shot by a content of the sequences. It should be noted that either if changes occur smoothly in the original video data, or if data are noisy, the procedures of the spatio-temporal segmentation do not always provide adequate results. 


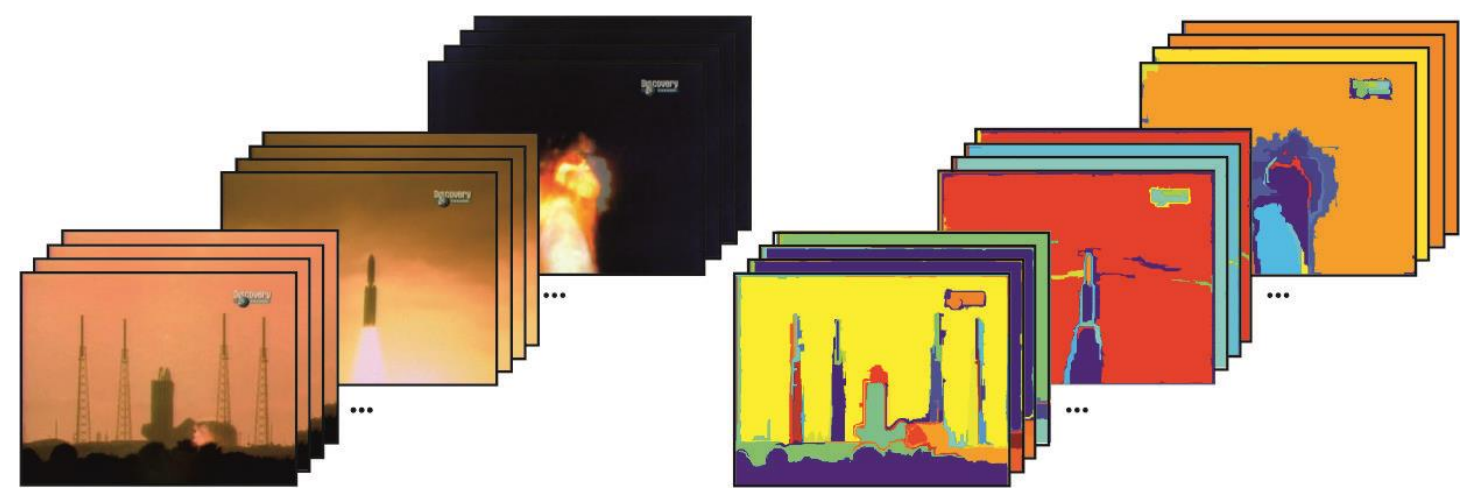

Fig.2. Examples of video sequences and the spatial segmentation of video frames

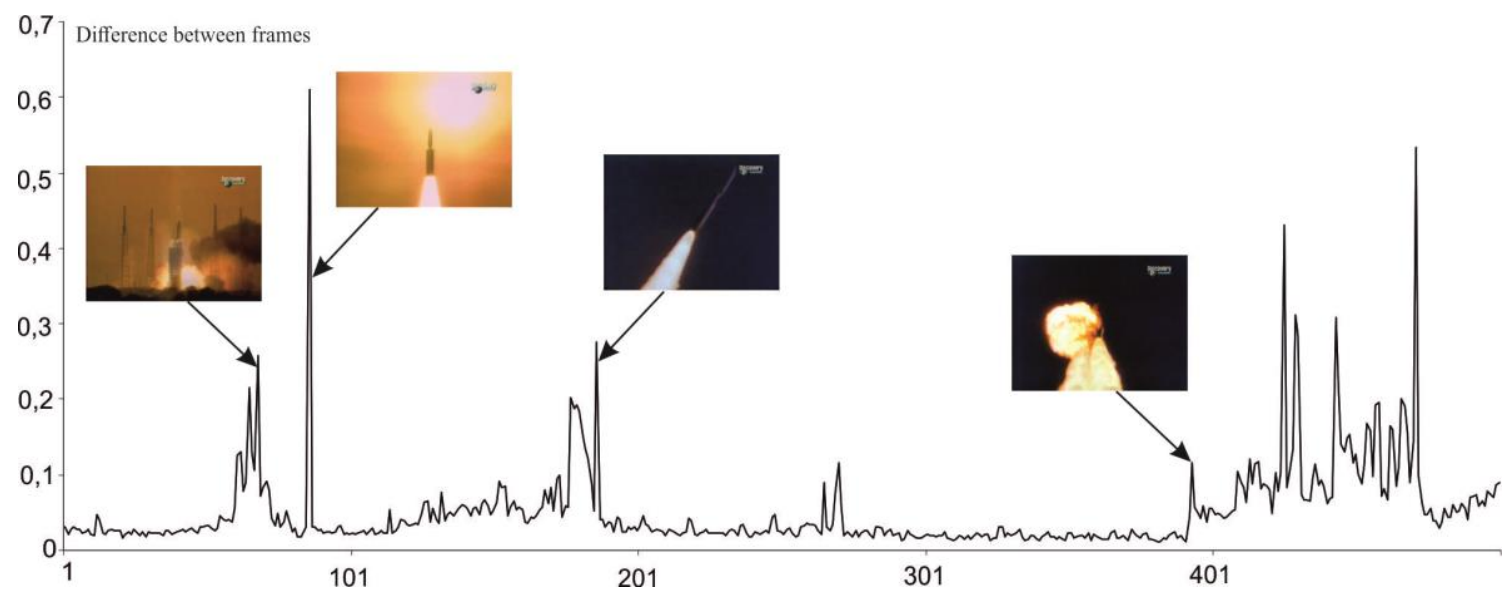

Fig.3. Differences between frames of a video sequence as result of the temporal segmentation

Further, to check the proposed method, a reduced video sequence was taken, for example, a moment of a catastrophe shot from several angles. An example of such a video sequence is shown in Fig.4. The IDTW method makes it possible to compare this video sequence of a different length with a set of video sequences from the database and to find the most relevant results after the clustering procedure has been applied. The results of the clustering/segmentation presented in Fig.5 demonstrate a correspondence level between this video and the one in Fig.3 (Fig.6). To be mentioned, the required result was obtained as a result of comparison of the video sets using the proposed modified IDTW procedure.

Since the comparison result cannot be a single video from the database, because it contains various videos of similar disasters, the next step was to test a number of video fragments with the video database using the developed IDTW clustering method. To evaluate the results obtained, a standard approach was used (also known as "Precision and recall"). This approach evaluates the correctness of the comparison results by finding a difference between relevant and irrelevant query results. Accordingly, these values are defined as

$$
P=\frac{t p}{t p+f p}, R=\frac{t p}{t p+f n}
$$

where $t p$ is a true positive result, $f p$ denotes a false positive result, and $f n$ stands for a false negative result. In this case, these values are not used in practical tasks and some measures created on their basis are used for evaluation of the final result. For example, the F-measure [43] (also called the harmonic mean) is calculated as

$$
F=2 \frac{P \cdot R}{P+R}
$$

This measure is approximately an average value of the precision and the recall when they are close enough.

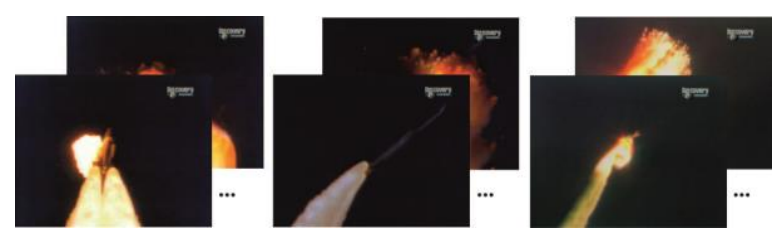

Fig.4. An example of a reduced video sequence

The results of the experiment for clustering 10 video fragments of different lengths and comparing them with the video base with further calculation of the described Fmetric are presented in Table 1. If the arithmetic average of the obtained results is then chosen, a value of the Fmeasure equals to 0.8707 . From the point of view of the information content, this result is quite satisfying for the clustering video sequences. 
Table 1. Results of clustering

\begin{tabular}{|c|c|}
\hline A video's number & F-measure \\
\hline 1 & 0.876 \\
\hline 2 & 0.922 \\
\hline 3 & 0.831 \\
\hline 4 & 0.915 \\
\hline 5 & 0.807 \\
\hline 6 & 0.862 \\
\hline 7 & 0.838 \\
\hline 8 & 0.946 \\
\hline 9 & 0.847 \\
\hline 10 & 0.863 \\
\hline & \\
\hline
\end{tabular}

At the same time, a rather vast range of values can be explained by several reasons. First of all, an amount of objects in the video database may not be sufficient that leads to roughened results in the precision and the recall. This fact is reflected by a distribution of the F-measure values. Secondly, the heterogeneity and ambiguity of videos while clustering data by the content adds some additional difficulties when it comes to searching of the similar data in terms of their characteristics. Thus, taking into account the indicated complexity, the results obtained are quite sufficient for being further applied as well as the given simplicity for implementation of the proposed modified iterative dynamic time warping approach for clustering matrix sequences.

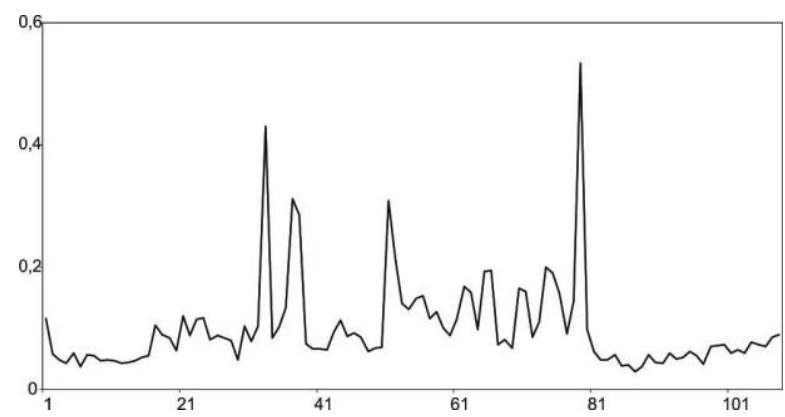

Fig.5. An example of clustering the reduced video sequence
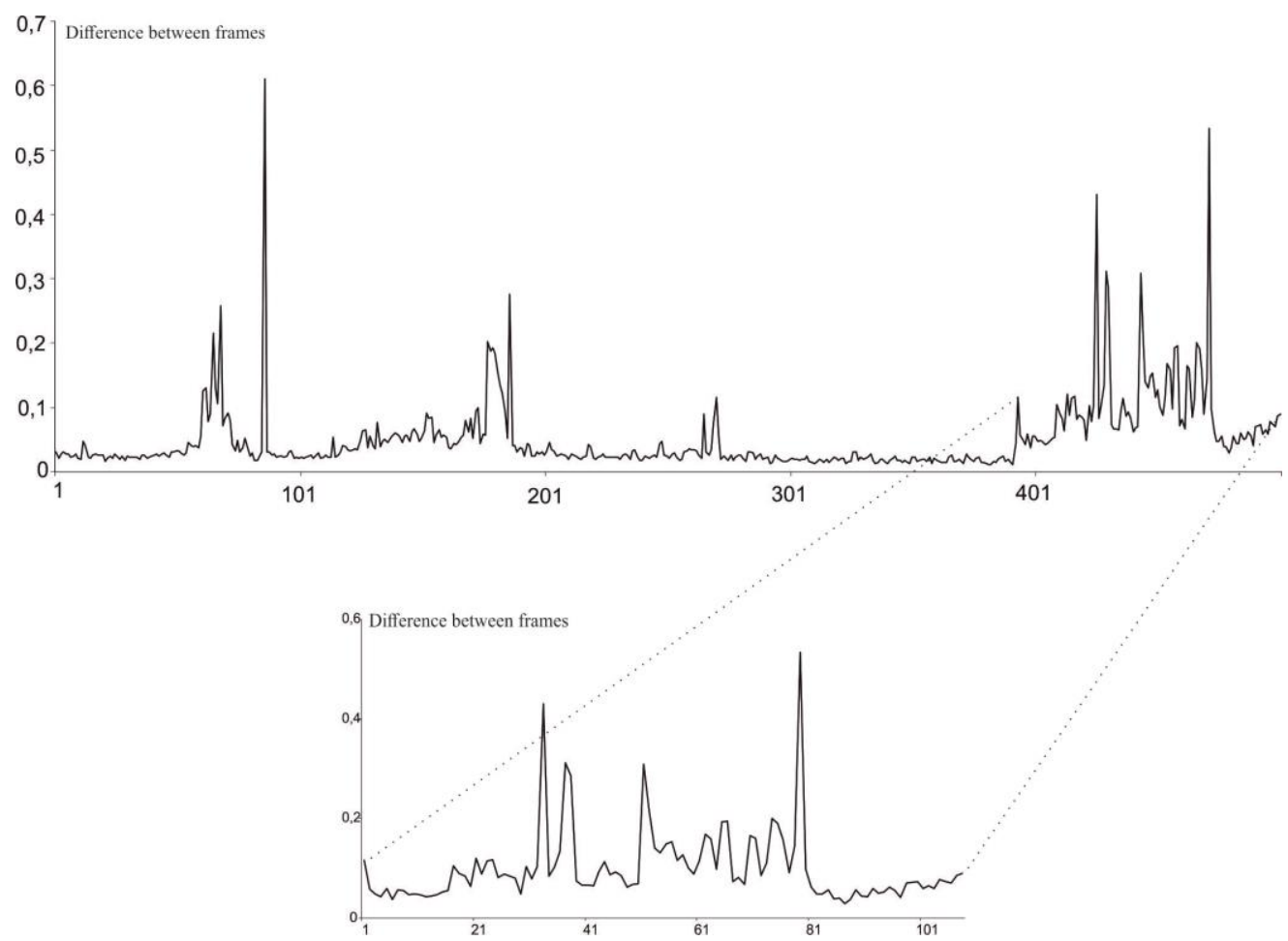

Fig.6. A correspondence level between two videos

\section{CONCLUSION}

The clustering method for the video sequences adapted from the modified Iterative Dynamic Time Warping technique and the succeeding clustering of reduced time series driven by the matrix fuzzy clustering method of the harmonic $k$-means is presented. A key feature of the developed approach is its plainness of numerical realization (compared to the prototype-based method), which is extremely important for processing video sequences.

\section{ACKNOWLEDGMENT}

This scientific work was financially supported by selfdetermined research funds of CCNU from the colleges' basic research and operation of MOE (CCNU16A02015). The third author also acknowledges the support of the Visegrad Scholarship Program-EaP \#51700967 funded by the International Visegrad Fund (IVF). 


\section{REFERENCES}

[1] D.P. Acharjya and V. Santhi, Bio-inspired computing for image and video processing. Boca Raton: CRC Press, 2018.

[2] H. Zhang, "Content-Based Video Analysis. Retrieval and Browsing", In: Feng D.D., Siu W.C., Zhang H.J. (eds) Multimedia Information Retrieval and Management. Signals and Communication Technology. Springer, Berlin, Heidelberg, pp. 27-56, 2003.

[3] V.S. Wold Eide, F. Eliassen, O.-C. Granmo, and O. Lysne, "Supporting timeliness and accuracy in distributed realtime content-based video analysis", Proc. of the eleventh ACM international conference on Multimedia, pp. 21-32, 2003.

[4] B. Huurnink, C.G.M. Snoek, M. de Rijke, and A.W.M. Smeulders, "Content-Based Analysis Improves Audiovisual Archive Retrieval", IEEE Transactions on Multimedia, vol. 14, no. 4, pp. 1166-1178, 2012.

[5] R. Nisbet, G. Miner, and K. Yale, Handbook of Statistical Analysis and Data Mining Applications, Second Edition. Elsevier, 2018.

[6] A.K. Jain and R.C. Dubes, Algorithms for Clustering Data. Englewood Cliffs, N.J.: Prentice Hall, 1988.

[7] L. Kaufman and P.J. Rousseeuw, Finding Groups in Data: An Introduction to Cluster Analysis. N.Y.: John Wiley \& Sons, Inc., 1990.

[8] R. Xu and D. Wunsch, Clustering. Wiley-IEEE Press, 2008.

[9] G. Gan, C. Ma, and J. Wu, Data Clustering: Theory, Algorithms, and Applications. Philadelphia: SIAM, 2007.

[10] J. Abonyi and B. Feil, Cluster Analysis for Data Mining and System Identification. Basel: Birkhäuser, 2007.

[11] C.C. Aggarwal and C.K. Reddy, Data Clustering: Algorithms and Applications. Boca Raton: CRC Press, 2014.

[12] Zh. Hu, Ye.V. Bodyanskiy, O.K. Tyshchenko, "A deep cascade neuro-fuzzy system for high-dimensional online fuzzy clustering",Proceedings of the 2016 IEEE 1st International Conference on Data Stream Mining and Processing (DSMP 2016), Lviv, Ukraine, pp. 318-322, 2016.

[13] S. Babichev, V. Lytvynenko, M. Korobchynskyi, M. Taif, "Objective clustering inductive technology of gene expression sequences features", Commun. Comput. Inf. Sci., No.716, pp.359-372, 2016.

[14] Zh. Hu, Ye.V. Bodyanskiy, O.K. Tyshchenko, "A cascade deep neuro-fuzzy system for high-dimensional online possibilistic fuzzy clustering", Proceedings of the 11th International Scientific and Technical Conference on Computer Sciences and Information Technologies, CSIT 2016, Lviv, Ukraine, pp. 119-122, 2016.

[15] Ye.V. Bodyanskiy, O.K. Tyshchenko, D.S. Kopaliani, "An Evolving Connectionist System for Data Stream Fuzzy Clustering and Its Online Learning", Neurocomputing, vol. 262, pp.41-56, 2017.

[16] Ye.V. Bodyanskiy, A.K. Tyshchenko, A.A. Deineko, “An evolving radial basis neural network with adaptive learning of its parameters and architecture", Automatic Control and Computer Sciences, vol. 49, iss. 5, pp. 255260, 2015.

[17] Zh. Hu, Ye.V. Bodyanskiy, O.K. Tyshchenko, O.O. Boiko, "A neuro-fuzzy Kohonen network for data stream possibilistic clustering and its online self-learning procedure", Applied Soft Computing Journal, vol. 68, pp. 710-718, 2018.
[18] S. Babichev, V. Lytvynenko, A. Gozhyj, M. Korobchynskyi, and M. Voronenko, "A Fuzzy Model for Gene Expression Profiles Reducing Based on the Complex Use of Statistical Criteria and Shannon Entropy", In: Hu Z., Petoukhov S., Dychka I., He M. (eds) Advances in Computer Science for Engineering and Education. ICCSEEA 2018. Advances in Intelligent Systems and Computing. Springer, Cham, vol.754, pp.545-554, 2019. DOI: https://doi.org/10.1007/978-3319-91008-6_55.

[19] J. Abonyi, B. Feil, S. Nemett, and P. Arva, "Fuzzy Clustering Based Segmentation of Time-Series", Advances in Intelligent Data Analysis V (IDA 2003). Lecture Notes in Computer Science, vol. 2810, pp. 275285, 2003.

[20] T.W. Liao, "Clustering of time series data", Pattern Recognition, vol.38, no.11, pp. 1857-1874, 2005.

[21] J. Han and M. Kamber, Data Mining: Concepts and Techniques. San Francisco: Morgan Kaufmann, 2006.

[22] D.L. Olson and D. Dursun, Advanced Data Mining Techniques. Berlin: Springer, 2008.

[23] C.C. Aggarwal, Data Mining. Cham: Springer, Int. Publ. Switzerland, 2015

[24] M.M. Deza and E. Deza, Encyclopedia of Distances. Dordrecht, Heidelberg, London, New York: Springer, 2009.

[25] X. Wang, A. Mueen, H. Ding, G. Trajcevski, P. Scheuermann, and E. Keogh, "Experimental comparison of representation methods and distance measures for time series data", Data Mining and Knowledge Discovery, vol.26, no.2, pp. 275-309, 2013.

[26] P.A. Jaskowiak, R.J. Campello, and I.G. Costa, "Proximity measures for clustering gene expression microarray data: a validation methodology and a comparative analysis", IEEE/ACM Trans Comput Biol Bioinform., vol.10, no.4, pp. 845-857, 2013.

[27] D. Kinoshenko, V. Mashtalir, and V. Shlyakhov, "A partition metric for clustering features analysis", International Journal "Information Theories and Applications", vol.14, iss.3, pp.230-236, 2007.

[28] S. Chu, E. Keogh, D. Hart, and M. Pazzani, "Iterative Deepening Dynamic Time Warping for Time Series", Proc. 2nd SIAM International Conference on Data Mining (SDM-02), 2002.

[29] N. Begum, L. Ulanova, J. Wang, and E. Keogh, "Accelerating dynamic time Warping Clustering with a Novel Admissible Pruning Strategy", Proc. of the 21th ACM SIGKDD International Conference on Knowledge Discovery and Data Mining, pp. 49-58, 2015.

[30] S. Salvador and P. Chan, "FastDTW: Toward Accurate Dynamic Time Warping in Linear Time and Space", KDD Workshop on Mining Temporal and Sequential Data, pp. 70-80, 2004.

[31] R.J. Kate, "Using dynamic time warping distances as features for improved time series classification", Data Mining and Knowledge Discovery, pp. 1-30, 2015.

[32] D.F. Silva and E.A.P.A.B. Batista, "Speeding up allpairwise dynamic time warping matrix calculation", http://sites.labic.icmc.usp.br/prunedDTW/, 2015.

[33] D.J. Berndt and S. Clifford, "Using Dynamic Time Warping to Find Patterns in Time Series", Proc. of the 3rd International Conference on Knowledge Discovery and Data Mining (AAAIWS'94), pp.359-370, 1994.

[34] E.J. Keogh and M.J. Pazzani, "Scaling up Dynamic Time Warping for Datamining Applications", Proc. of the 6th ACM SIGKDD International Conference on Knowledge Discovery and Data Mining, pp. 285-289, 2000. 
[35] E.J. Keogh and M.J. Pazzani, "Derivative Dynamic Time Warping", Proc. of the First SIAM International Conference on Data Mining (SDM'2001), 2001.

[36] T. Rakthanmanon, B. Campana, A. Mueen, G. Batista, B. Westover, Q. Zhu, J. Zakaria, and E. Keogh, "Searching and Mining Trillions of Time Series Subsequences under Dynamic Time Warping", Proc. of the 18th ACM SIGKDD International Conference on Knowledge discovery and data mining (KDD'12), pp. 262-270, 2012.

[37] S. Mashtalir, V. Mashtalir, and M. Stolbovyi, "Video Shot Boundary Detection Via Sequential Clustering", International Journal "Information Theories and Applications", Vol.24, No.1, pp.50-59, 2017.

[38] C. Faloutsos, M. Ranganathan, and Y. Manolopoulos, "Fast subsequence matching in time-series databases", Proc. ACM SIGMOD International Conference on Management of Data, 1994.

[39] Zh. Hu, S.V. Mashtalir, O.K. Tyshchenko, M.I. Stolbovyi, "Video Shots' Matching via Various Length of Multidimensional Time Sequences", International Journal of Intelligent Systems and Applications (IJISA), Vol.9, No.11, pp.10-16, 2017.

[40] I.V. Nikiforov, "Sequential Detection of Changes in Stochastic Process", IFAC Proceedings Volumes, vol.25, iss.15, pp.11-19, 1992.

[41] B. Zhang, M. Hsu, and U. Dayal, "K-Harmonic Means -A Spatial Clustering Algorithm with Boosting", In: Roddick J.F., Hornsby K. (eds) Temporal, Spatial, and SpatioTemporal Data Mining. Lecture Notes in Computer Science, vol. 2007, pp.31-45, 2001.

[42] W.-C. Yeh, Y. Jiang, Y.-F. Chen, and Z. Chen, "Correction: A New Soft Computing Method for KHarmonic Means Clustering”, PLoS ONE 12(1), 2017. https://doi.org/10.1371/journal.pone.0169707.

[43] N. Chinchor, "MUC-4 Evaluation Metrics", Proc. of the Fourth Message Understanding Conference, pp. 22-29, 1992.

\section{Authors' Profiles}

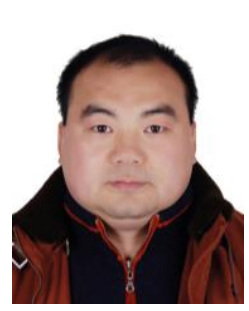

Zhengbing Hu: Ph.D., Associate Professor of School of Educational Information Technology, Central China Normal University, M.Sc. (2002), Ph.D. (2006) from the National Technical University of Ukraine "Kiev Polytechnic Institute". Postdoc (2008), Huazhong University of Science and Technology, China. Honorary Associate Researcher (2012), Hong Kong University, Hong Kong. Major interests: Computer Science and Technology Applications, Artificial Intelligence, Network Security, Communications, Data Processing, Cloud Computing, Education Technology.

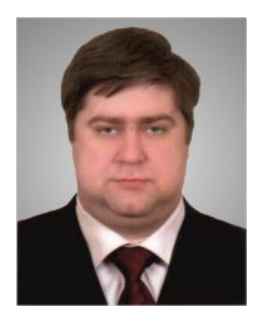

Sergii Mashtalir graduated (M.Sc.) from Kharkiv National University of Radio Electronics (KhNURE) in 2001. He got his $\mathrm{PhD}$ in 2005 and Dr. Habil. Sci. Eng. in 2016.

He is currently working as Professor of Informatics Department at KhNURE. He has about 100 scientific publications including 2 monographs. His research interests are image processing and recognition, video parsing, content based image and video retrieval.

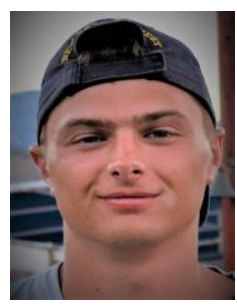

Oleksii K. Tyshchenko got his MSc from Kharkiv National University of Radio Electronics in 2008. He got his $\mathrm{PhD}$ in Computer Science in 2013. He is currently working as Senior Researcher at Control Systems Research Laboratory, Kharkiv National University of Radio Electronics, Kharkiv, Ukraine and holds a postdoc scholar position at Institute for Research and Applications of Fuzzy Modeling, CE IT4Innovations, University of Ostrava, Ostrava, Czech Republic. He has currently published more than 70 publications. He is a reviewer of such journals as Neural Computing and Applications (NCAA); Soft Computing (SoCo); Evolving Systems (EvoS); Neurocomputing (Neurocomp); Expert Systems With Applications (ESWA); IEEE Transactions on Cybernetics; IEEE Transactions on Fuzzy Sets and Systems; Fuzzy Sets and Systems; Pattern Recognition Letters.

His current research interests are Evolving Cascade NeuroFuzzy Systems; Computational Intelligence; Machine Learning; Deep Learning; High-Dimensional Fuzzy Clustering.

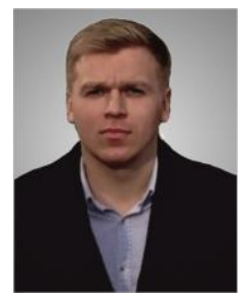

Mykhailo Stolbovyi graduated from Kharkiv National University of Radio Electronics in 2007. He is a PhD student in Computer Science at Kharkiv National University of Radio Electronics. His current interests are image and video processing, video clustering, and computational intelligence.

How to cite this paper: Zhengbing $\mathrm{Hu}$, Sergii V. Mashtalir, Oleksii K. Tyshchenko, Mykhailo I. Stolbovyi, "Clustering Matrix Sequences Based on the Iterative Dynamic Time Deformation Procedure", International Journal of Intelligent Systems and Applications(IJISA), Vol.10, No.7, pp.66-73, 2018. DOI: 10.5815/ijisa.2018.07.07 